\title{
Photoprotection and growth under different lights of Arabidopsis single and double mutants for energy dissipation (npq4) and state transitions (pph1)
}

Thi Thu Hương Khuong ${ }^{1,2,3^{*}}$, Christophe Robaglia ${ }^{1}$, Stefano Caffarri $^{1^{*}}$

${ }^{1}$ Aix Marseille Université, CEA, CNRS, Biosciences and Biotechnologies Institute of Aix-Marseille (BIAM), Equipe de Luminy de Génétique et Biophysique des Plantes, 13009 Marseille, France.

${ }^{2}$ Cell Technology Laboratory - CFB - Vietnam National University of Forestry, Hanoi, Vietnam.

${ }^{3}$ The Key Laboratory of Enzyme and Protein Technology (KLEPT), Hanoi University of Science (HUS), Vietnam National University (VNU), Hanoi, Vietnam.

*Corresponding authors:

stefano.caffarri@univ-amu.fr, Tel: +33 4 91829562, ORCID 0000-0002-4729-7679

huongktt@vnu.edu.vn, Tel ++8402435575494

\begin{abstract}
Non Photochemical Quenching (NPQ) is a short-term regulation important to maintain efficient photosynthesis and to avoid photooxydative damages by dissipation of excess energy. Full activation of NPQ in plants requires the protonation of the PsbS protein, which is the sensor of the low lumenal $\mathrm{pH}$ triggering the thermal dissipation. State transitions are a second important photosynthetic regulation to respond to changes in light quality and unbalanced excitation of photosystems. State transitions allow energy redistribution between PSI and PSII through the reversible exchange of LHCII antenna complexes between photosystems thank to the opposite action of the STN7 kinase and PPH1 phosphatase: phosphorylation of LHCII promotes its mobilization from PSII to PSI, while dephosphorylation has the opposite effect. In this work, we produced the pph1/npq4 double mutant and characterized some photosynthetic, growth and reproduction properties in comparison with wild type and single mutant plants in high and low light conditions. Results indicate that in high light, the pphl mutant maintains good photoprotection ability, while $n p q 4$ plants show more susceptibility to photodamages. The $p p h 1 / n p q 4$ double mutant showed a resistance to high light stress similar to that of the single $n p q 4$ mutant. In low light condition, the single mutants showed a significant increase of growth and flowering as compared to wild type plants and this effect was further enhanced in the pph1/npq4 double mutant. Results suggest that photosynthetic optimisation to improve crop growth and productivity might be possible, at least under controlled low light conditions, by modifying NPQ and state transitions regulations.
\end{abstract}

Key word: Non-photochemical quenching, PsbS protein, PPH1/TAP38 protein, plant growth, state transitions.

Key Message: Arabidopsis single and double mutants for energy dissipation (npq4) and state transition (pphl, blocked in state II) show enhanced growth and flowers+siliques production under controlled low light conditions. 


\section{Introduction}

During photosynthesis, light is absorbed by chlorophyll and carotenoid pigments, mainly localized in antenna proteins, and excitation energy is transferred to reaction centers where charge separation and electron transfer occur. Light is therefore indispensable for survival, but plants need to cope with different environmental situations where light quantity and quality can be not optimal for photosynthesis. If absorbed energy is more than the quantity exploitable by plant metabolism, this can lead to a variety of harmful consequence for plant and in particular to the production of reactive oxygen species (ROS) that can impair photosynthesis and diminish plant growth (Melis 1999). A major source of ROS in plants is the chlorophyll in the triplet excited state formed by inversion of the spin of a singlet excited chlorophyll which is not utilized for photochemistry, especially in situation of photosynthesis saturation (Krieger-Liszkay 2005). Energy of triplet state chlorophylls can be transferred to oxygen molecules to generate singlet excited oxygen, a very harmful ROS (Krieger-Liszkay 2005).

To avoid ROS formation, Non Photochemical Quenching (NPQ) dissipates excess energy as heat. NPQ is considered a short term regulation important for maintaining efficient photosynthesis and avoid photo-oxydative damages in high or fluctuating light (Kulheim et al. 2002; Krah and Logan 2010; Hubbart et al. 2012). The steadystate redox level of the primary quinone acceptor $\left(\mathrm{Q}_{\mathrm{A}}\right)$ of PSII is a determinant parameter to have efficient photochemistry or photodamages under a variety of physiological and environmental conditions (Melis 1999). When $\mathrm{Q}_{\mathrm{A}}$ is reduced, PSII reaction center is closed, and excitation energy at PSII must be dissipated to avoid photodamages. On the contrary, when $\mathrm{Q}_{\mathrm{A}}$ is oxidized, excitation energy can be efficiently utilized in photochemical reactions and electron transport. Under steady-light conditions, the reduction state of $\mathrm{Q}_{\mathrm{A}}$ increases gradually with irradiance, therefore causing a correspondingly increase in the probability of photodamage (Melis 1999).

In recent years, numerous researches focused on NPQ mechanism (reviewed in (Muller et al. 2001; Li et al. 2009; Gorbunov et al. 2011; Wilhelm and Selmar 2011; Ruban et al. 2012)). Nevertheless, the precise mechanism is still unclear. It is known that full activation of qE, which is the major and fastest component of NPQ, requires a low lumenal $\mathrm{pH}$, the synthesis of zeaxanthin through the activation of the xanthophyll cycle, the protonation of the PsbS protein and the participation of the Lhcb proteins of PSII (Ruban et al. 2012). PsbS plays the key role of sensor of low lumenal pH thanks to two lumenal protonable glutamates ( $\mathrm{Li}$ et al. 2000; Li et al. 2002b; Li et al. 2004). However how PsbS can activate $\mathrm{qE}$ after protonation is still unclear, although PsbS properties have been investigated in many papers since long time (Muller et al. 2001; Li et al. 2002b; Li et al. 2004; Horton and Ruban 2005; Kalituho et al. 2006; Bonente et al. 2008; Kiss et al. 2008; Li et al. 2009; Johnson and Ruban 2010; Kereiche et al. 2010; Bonente et al. 2011; Gorbunov et al. 2011; Johnson and Ruban 2011; Kasajima et al. 2011; Wilhelm and Selmar 2011; Ruban and Murchie 2012; Niyogi and Truong 2013; Sylak-Glassman et al. 2014; Dong et al. 2015; Tibiletti et al. 2016; Ruban 2017; Sacharz et al. 2017; Głowacka et al, 2018).

It has been proposed that PsbS itself is the site of energy quenching (Niyogi et al. 2005); however, because PsbS does not seem to bind pigments as other proteins of the Lhc family (Funk et al. 1995; Dominici et al. 2002; Bonente et al. 2008), a property necessary to catch and quench excitation energy, it is probable that PsbS is not the quencher. Various results indicate that PsbS would be involved in the reorganization of the thylakoid macrostructure towards a quenching state triggering energy dissipation (Horton et al. 2005; Kiss et al. 2008; Betterle et al. 2009; Kereiche et 
al. 2010). Thus, quenching would occur inside the Lhc complexes by conformational change of these proteins (Ruban et al. 2007; Ahn et al. 2008; Holzwarth et al. 2009).

A second important photosynthetic regulation to respond to changes in light intensity and quality is called "state transitions" (Bonaventura and Myers 1969; Murata 1969; Rochaix 2014). State transitions are a mechanism by which excitation energy is redistributed between PSI and PSII when plants are exposed to lights that preferentially excite either PSI or PSII (Haldrup et al. 2001). Indeed absorption of PSI and PSII is different due to their different pigment-protein composition (Veeranjaneyulu and Leblanc 1994). State transitions are induced by the redox state of the plastoquinone pool and the Cyt $b_{6} f$ complex (Wollman 2001; Mao et al. 2002; Lemeille et al. 2009; Minagawa 2011; Puthiyaveetil et al. 2012). Redistribution of excitation energy between photosystems requires the reversible movement of LHCII, the major antenna protein of PSII, between PSII and PSI. The STT7 and STN7 kinase proteins in green algae and plants respectively (Depege et al. 2003; Bellafiore et al. 2005), and the plant PPH1 phosphatase protein (also called TAP38) have a fundamental role in state transitions (Pribil et al. 2010; Shapiguzov et al. 2010). In plants, under illumination conditions that are favorable for PSII excitation (red/blue light), the plastoquinone pool (PQ) becomes more reduced, the STN7 kinase is activated and phosphorylates LHCII, part of which migrates to PSI (State II condition). Under light conditions that preferentially excite PSI (as far red light), PQ is oxidized, STN7 kinase is inactivated, LHCII dephosphorylation is catalyzed by the PPH1 phosphatase and LHCII returns to PSII (State I). Mobile antennas between PSII and PSI represent about 20-25\% of the total pool of LHCII in higher plants (Allen 1992), mainly represented by LHCII loosely bound to PSII (Galka et al. 2012). This reversible redistribution exhibits kinetics for induction/relaxation of about 15 to $30 \mathrm{~min}$ (Haldrup et al. 2001; Allen 2003).

The PPH1 phosphatase responsible for the dephosphorylation of LHCII (Pribil et al. 2010; Shapiguzov et al. 2010) is a thylakoid-associated phosphatase of $38 \mathrm{kDa}$. In pph1 Arabidopsis plants inactivated for the PPH1 function, PLHCII are not dephosphorylated and thylakoids are blocked in the so-called "State II", enriched in PSI-LHCII complexes (Pribil et al. 2010; Shapiguzov et al. 2010). It has been also shown that the pph1 mutants have a particular and unexpected growth phenotype in low light. Indeed it has been reported that LHCII hyperphosphorylation due to inactivation of PPH1 could improve growth under low light conditions (Pribil et al. 2010). Physiological importance of state transitions on plant growth and fitness has been well demonstrated in the presence of the $\sin 7$ mutation (Tikkanen et al. 2010), which block thylakoids in State I, while less physiological characterisation of the $p p h 1$ mutant (blocked in State II) is available.

In the context of the study of photosynthetic regulation by energy quenching (NPQ) and state transitions, we produced the $p p h 1 / n p q 4$ double mutant by crossing $n p q 4$ and $p p h 1$ Arabidopsis mutants. In this paper, we report results on photosynthetic properties in low light and high light and on growth and reproduction in low light of the pph1/npq4 double mutant in comparison with single mutants and wild type plants. 


\section{Materials and methods}

\section{Plant growth conditions}

Low light (LL) growth experiments were performed in a growth chamber with $\sim 20 \mu \mathrm{mol} \mathrm{m} \mathrm{m}^{-2} \mathrm{~s}^{-1}$ homogenous illumination; normal light (NL) was set at $\sim 120 \mu \mathrm{mol} \mathrm{m}^{-2} \mathrm{~s}^{-1}$; long day (16 hr light $/ 8 \mathrm{hr}$ dark) and $22^{\circ} \mathrm{C}$ were set both for LL and NL conditions. The spectra of the light sources are shown in supplemental information (Fig. S1).

\section{Creation of the pph1/npq4 double mutant}

To produce the $p p h 1 / n p q 4$ double mutant, we crossed pollen of pph1.3 (GABI_232H12, Col-0 ecotype) (Alonso et al. 2003; Shapiguzov et al. 2010) with ovule of $n p q 4.1$ (Col-0 ecotype) (Li et al. 2000). The sterilized F1 seeds were grown on the MS medium plates with sulfadiazine (5 mg/l), carried in the T-DNA used in the GABI lines (Awan et al. 2008). 10 days-F2 plantlets surviving on medium containing sulfadiazine were screened firstly for NPQ phenotype ( $p s b S$ mutation) by video imaging of chlorophyll fluorescence, similarly as in (Niyogi et al. 1998). Putative double mutants were screened a second time for NPQ and by fluorescence at $77 \mathrm{~K}$ to detect plants blocked in State II (Pribil et al. 2010; Shapiguzov et al. 2010). Far-red light was used to induce State I (Philips E27PF712E, a red darkroom lamp). Leaves were grinded in a buffer containing $10 \mathrm{mM}$ Hepes-KOH, $\mathrm{pH} 7.5$ and 100\% (w/v) of glycerol. Low-temperature $(77 \mathrm{~K})$ fluorescence emission spectra were recorded on this extract in liquid nitrogen using a Cary Eclipse spectrofluorometer. Excitation was at $475 \mathrm{~nm}$ (slit width $2.5 \mathrm{~nm}$ ) and emission was recorded in the $600-800 \mathrm{~nm}$ range (slit width $2.5 \mathrm{~nm}$ ). F3 plants from the putative F2 double mutant plants were confirmed by comparison of NPQ and low temperature fluorescence emission spectra with single mutants of $n p q 4, p p h 1$ and wild type Col-0 ecotype (Fig. 1a and b). The presence of PsbS and PPH1 proteins was checked by immunoblot analysis using antibodies directed against PsbS (Tibiletti et al. 2016) and against PPH1 (antibody raised against the peptide (C)TKKNDMLKKGVDEG). Homozygote F3 plants were used in the following experiments.

\section{Plant growth, flower/silique production and light stresses}

Seeds used for growth experiments were harvested for each genotype from plants grown in the same conditions $\left(22^{\circ} \mathrm{C}, 16 \mathrm{hr}\right.$ light, $120 \mu \mathrm{mol}$ photons $\left.\mathrm{m}^{-2} \mathrm{~s}^{-1}\right)$. Seed had similar germination rate, and identical germination time and initial growth (by visual inspection). Plant growth at advanced stages was determined as dry weight of the rosette of plants grown 17 days in normal light and then 20,33, 37 days in low light for test 1, test 2 and test 3, respectively (Fig. 3). Siliques and flowers were counted on plants grown 17 days in normal light then 24, 37 days in low light, indicated as test 1 and test 2 in figure 6. High light stresses were performed using cool-white LED lights (Fytoled, Photon System Instruments), which provide uniform vertical light. Thanks to the use of LED sources and large chambers (several cube meters), no significant change of the temperature was present on a single plant or between plants during high light treatments. For all experiments, plants were randomized during treatments.

\section{Pigment analysis}

Frozen leaves were grinded in a mortar and pigments extracted by repeated washing with acetone $90 \%$ buffered with $\mathrm{Na}_{2} \mathrm{CO}_{3}$. Extracts were diluted to $80 \%$ acetone and analysed for total chlorophylls content and $\mathrm{Chl} /$ carotenoid ratio by fitting of the absorption spectra (Croce et al. 2002) taken with a Cary300 spectrophotometer, and by high 
performance liquid chromatography (Campoli et al. 2009), which allows the precise estimation of single carotenoid content.

\section{Fluorescence analysis}

PAM measurements were performed using a dual PAM-F (Walz) or an imaging fluorometer (Fluorcam FC 800-O; Photon System Instruments) at room temperature and using standard procedures. The maximum PSII quantum yield and the actual PSII quantum yield during a light period were measured accordingly to the equations: $\mathrm{Fv} / \mathrm{Fm}=(\mathrm{Fm}$ $\mathrm{Fo}) / \mathrm{Fm}$ and $\Phi P S I I=\left(\mathrm{Fm}^{\prime}-\mathrm{Ft}\right) / \mathrm{Fm}$ ' (Genty et al. 1989). Fm is the maximum fluorescence yield and Fo is the minimal fluorescence yield of plants dark adapted for 30-min at room temperature; Fm' is maximum fluorescence yield of light adapted plants and Ft is the steady state fluorescence yield under actinic light. NPQ was calculated as $\left(\mathrm{Fm}-\mathrm{Fm}^{\prime}\right) / \mathrm{Fm}$ '. State transitions have been measured using the imaging fluorometer as described in (Crepin et al. 2015).

\section{Statistical analysis}

Statistical analysis was performed using the Student's t-test and differences were considered significant when the pvalue was less than 0.05 . Details about sample size and specific conditions for measurements are provided in the legend of the figures and tables.

\section{Results and discussion}

\section{Isolation of the pph1/npq4 double mutant}

The double pph1/npq4 mutant was screened on the F2 seedling using the typical phenotypes of the single parental mutants: NPQ decrease of the npq4 mutant (Li et al. 2000) and an elevated PSI fluorescence emission at $77 \mathrm{~K}$ in the pph1 mutant as compared to wild type plants after a preliminary exposure to PSI light (far red) to induce State I (Pribil et al. 2010; Shapiguzov et al. 2010). The detection of the $p p h 1$ mutation was also facilitated by the survival of plants on a medium containing sulfadiazine (the marker on the T-DNA of the GABI collection used to isolate the pph1 mutant).

F3 plants obtained from putative homozygote $p p h 1 / n p q 4$ double mutant plants were further checked for the presence of the double mutation using several phenotypic traits (Fig. 1). The NPQ decrease was clearly observed in pph1/npq4 double mutants as compared with wild type plants and was similar to npq4 mutants (Li et al. 2000) (Fig. 1a). The constitutive associations of phosphorylated LHCII with PSI (State II) in mutant plants has been confirmed by the relative increase of PSI to PSII cross-section by measurement of low temperature fluorescence emission spectra, since PSI and PSII emissions are well separated (peak at 734 and 684 nm, respectively; Fig. 1b). A significant emission of PSI fluorescence was observed in $p p h 1$ and in the double mutants as compared with wildtype and $n p q 4$ plants indicating that PSI-LHCII complexes were always persisting in these mutants, but not in $n p q 4$ and in wild type plants. The absence of the PsbS and PPH1 proteins was confirmed by immunoblot by using specific antibodies (Fig. 1c). Finally, the absence of state transition was confirmed by using imaging PAM fluorescence techniques (Fig. 1d and supplemental Fig. S2). We concluded that both $p s b S$ and $p p h 1$ are mutated in our selected putative double mutants. We also did not detect any significant influence of a single mutation (npq4 or pphl) on 
phenotypic traits associated with the second gene (respectively, state transition capability/PPH1 accumulation and NPQ induction/PsbS accumulation, Fig. 1b-c-d and supplemental Fig. S2). This suggests that, at least for the phenotypes investigated, there are no evident interactions between photosynthesis regulations controlled by PsbS and PPH1.

\section{Response of mutants lacking PsbS and PPH1 to high light stress}

We estimate the photoprotective ability of the double mutant and the single mutants in comparison with wild type plants during a high light stress. Under high light, the reaction centers of photosystems become progressively saturated resulting in a decrease of energy utilization in photosynthesis and a subsequent increase of "unused" harmful excitation energy in the photosynthetic membrane. This effect is particularly evident on Photosystem II and it can be observed as a change in PSII chlorophyll fluorescence properties. To this aim, we measured the Fv/Fm parameter, which provides the maximum quantum yield of PSII photochemistry, an important physiological indicator of the state of the photosynthetic apparatus in intact plant leaves (Genty et al. 1989).

Plants grown under normal light have been treated with $1500 \mu \mathrm{mol} \mathrm{m}^{-2} \mathrm{~s}^{-1}$ at $\sim 12^{\circ} \mathrm{C}$ (Fig. 2). Fv/Fm measured before stress was the same for all genotypes, accordingly to previous publications on single mutants (Li et al. 2000; Pribil et al. 2010). After a 10 hours treatment under high light (Fig. 2), Fv/Fm of all genotypes measured after 30 minroom temperature dark-adaptation decreased as compared with values before stress indicating that PSII was photoinhibited during the high light stress. The decrease of Fv/Fm (Fig. 2) was higher in the $n p q 4$ mutant and in the pph1/npq4 double mutant, which had similar values, while WT and pph1 mutant plants had similar Fv/Fm and thus were less photoinhibited than $n p q 4$ and $p p h 1 / n p q 4$ plants. Similar conclusions have been obtained in experiments performed at $22^{\circ} \mathrm{C}$ (supplemental Fig. S4). This indicates that a higher photoinhibition under constant high light is associated only with the $n p q 4$ mutation, while the $p p h 1$ mutation has a negligible effect on the photoprotective capability under these conditions.

This is consistent with the fact that a reduced thermal dissipation (NPQ) in mutants lacking PsbS (Li et al. 2000; Li et al. 2002b) leads at high light intensities to overexcitation and damage of PSII. The result supports also that state transitions are an important regulative mechanism under non saturating light conditions, while they are inhibited under high light (Rintamaki et al. 1997; Rintamaki et al. 2000; Tikkanen et al. 2008; Tikkanen et al. 2010). Indeed dissipation of excess energy (NPQ) is more important under high light than the control of relative absorption of photosystems (state transitions). Our results also show that the absence of PsbS has an impact during a short high light stress both when plants are in State I (as in the case of the $n p q 4$ single mutant) or in State II (as in the case of the $p p h 1 / n p q 4$ double mutant, which is blocked in State II).

It should be noted that the pph1 mutant has a PSI antenna size increased as compared to control plants even under a short high light stress (Mekala et al. 2015) as well as after several hours under high light (supplemental Fig. S5). However, even if not directly measured in our work, PSI does not seem more damaged in the $p p h 1$ mutants as compared to the respective control plants under high light. If it was the case, impaired electron transport at PSI would also cause some damage to PSII, an effect that has not been detected in our experiments (Fig. 2 and supplemental Fig. S4). PSI is usually able to efficiently dissipate excess energy and significant photoinhibition can 
be detected only in conditions of acceptor side limitation (Sonoike 2011). Indeed PSI photoinhibition is particularly visible at temperature lower than $10^{\circ} \mathrm{C}$ in chilling-sensitive plants as cucumber (Sonoike et al. 1995) and even in cold-resistant plants as barley and Arabidopsis at $4^{\circ} \mathrm{C}$ (Tjus et al. 1998; Zhang and Scheller 2004). In these conditions a decreased activity of the enzymes of the Calvin cycle and of the water-water cycle causes a nonutilization of reduced PSI acceptors (Ferredoxin) with a consequent over-reduction of the Fe-S clusters in PSI, a subsequent formation of reactive oxygen species and finally damages to PSI. A similar PSI photoinhibition can be obtained in mutants impaired in cyclic and pseudo-cyclic electron flows, which, in WT plants, allow alleviating PSI acceptor side limitations and sustain NPQ activation by decreasing luminal pH (Asada 2002; Munekage et al. 2004; Suorsa et al. 2012; Gerotto et al. 2016; Shikanai and Yamamoto 2017). Therefore, it is evident than in the conditions used in our experiments (constant high light at $12^{\circ} \mathrm{C}$, Fig. 2 , or $22^{\circ} \mathrm{C}$, Fig. S4), additional LHCII attached to PSI in the pph1 mutants does not cause any particular damage to PSI. In these conditions, cyclic and alternative electron pathways seems sufficient for protecting PSI in pph1 plants in a similar way as in WT plants.

At the same time, the decreased antenna size of PSII in the pphl does not cause a reduced photoinhibition of this photosystem under high light (Fig. 2 and supplemental Fig. S4). It should be noted however that only a relatively small fraction of LHCII ( 20\%) would associate to PSI in State II (Allen 1992; Galka et al. 2012). Moreover, NPQ capacity, which depends on several factors (in particular on PsbS and zeaxanthin amounts and on luminal $\mathrm{pH}$ ), is similar in pph1 and WT plants (Fig. 1), which allows an equivalent PSII photoprotection in both genotypes.

It can be concluded that state transitions do not have a positive effect on photoprotection under constant high light, but neither a negative impact. However, as previously suggested (Mekala et al. 2015), it is likely that under fluctuating light, the slowly reversible state transitions might have a negative impact during a shift from high to low light because of excitation imbalances between photosystems. For this reason plant would avoid state transitions under high light (Rintamaki et al. 1997; Mekala et al. 2015).

\section{Pigment composition change of mutants lacking PsbS and PPH1 under different light conditions}

Chlorophylls and carotenoids are responsible for absorption of light and transfer of excitation energy to reaction centres for photochemistry. Carotenoids have also an important role in photoprotection during a light stress. Therefore determinations of leaf pigment content can provide valuable information about photosynthetic complexes in mutants or during a stress. Moreover, the complete activation of NPQ requires not only the protonation of PsbS ( $\mathrm{Li}$ et al. 2000; Dominici et al. 2002; Li et al. 2004), but also the synthesis of zeaxanthin during the xanthophyll cycle operations (Demmig-Adams 1990). Zeaxanthin free in the membrane has also a role in photoprotection independent from NPQ, being an efficient scavenger of ROS (Havaux and Niyogi 1999). Thus, to investigate the acclimation response to different light conditions, pigments from leaves of mutants and wild type plants grown in normal light, low light and treated under high light were extracted and analyzed by HPLC and spectrophotometry (Table 2).

Pigments from leaves acclimated at low light and normal light were similar between the four genotypes (Table 1). In particular under low light and in normal light, mutant and wild type plants (Table 1) had similar Chl $a / b$ ratios of 3.0. Similar pigment composition between wild type and $n p q 4$ was already reported (Li et al. 2002a). However, 
after high light treatment, plants exhibited a change in chlorophyll and carotenoid pigment content. $\mathrm{Chl} a / b$ ratio reflects a change in the supercomplex structure. It is indeed well known that antenna complexes are reduced under high light, and indeed an increase in the $\mathrm{Chl} a / b$ ratio indicates a reduction of Lhc antenna (rich in $\mathrm{Chl} b$ ) relatively to the core complex (lacking $\mathrm{Chl} b$ ). The change of $\mathrm{Chl} a / b$ ratio in the $n p q 4$ mutant was similar to that of wild type plants, which could reflect a similar acclimation to high light when the stress is prolonged (Golan et al. 2006). This ratio was particularly elevated in the $p p h 1$ single mutant, but not in the double $p p h 1 / n p q 4$ mutant. This point needs further analysis to understand the molecular basis at the origin of this particularly high $\mathrm{Chl} a / b$ ratio in the single pph1 mutant and not in the double mutant.

Carotenoids bound to proteins or free in membrane play important roles for photoprotection in plants. In low light, we did not find statistical significant differences in carotenoids content between mutants and wild type, as well as between the double mutant and simple mutants. Under high light some differences have been detected. Total carotenoid amount increased on a Chls basis (i.e. Chls/Cars ratio decreased) in all genotypes after 4.5 days of treatment at $1600 \mu \mathrm{mol} \mathrm{m} \mathrm{m}^{-2}$ as compared with plants in low and normal light. However, in the absence of PPH1 (single and double mutant) the increase of carotenoids was particularly high as compared with that of $n p q 4$ and of wild type plants $(25-40 \%$ higher) (Table 1$)$, and the carotenoids of the xanthophyll cycle, violaxanthin (Vio), antheraxanthin (Ant) and zeaxanthin (Zea), displayed the highest increase (35-55\%). These carotenoids are involved in the process of non-photochemical quenching of the absorbed energy (Demmig-Adams 1990) and other photoprotective mechanisms (Havaux and Niyogi 1999). The xanthophyll cycle consists in the reversible deepoxidation of violaxanthin to zeaxanthin via the intermediate antheraxanthin (Demmig-Adams 1990). The deepoxidation takes place when the violaxanthin de-epoxidase is activated by a low lumen $\mathrm{pH}$ due to the formation of a high transmembrane proton gradient during high illumination (Gilmore and Yamamoto 1992). However, despite higher amounts of the xanthophyll cycle carotenoids (VAZ=Vio+Ant+Zea) in the presence of the pph1 mutation, the de-epoxidation index ((0.5Ant+Zea)/VAZ)) was similar between the four genotypes.

In addition, also the other carotenoids (neoxanthin, lutein and $\beta$-carotene) increased in mutants carrying the pph1 mutation as compared with the wild type under high light. Notably, the highest carotenoid increased (i.e. the lowest Chls/Cars ratio) was found in the double mutant. In conclusion, under non-stressing condition, all genotypes had a similar carotenoid content, while under high light the npq4 mutant showed a very small increase (but statistically not significant in our analysis) in total carotenoid content as compared with the wild type, the mutant lacking PPH1 showed a stronger effect and the double mutant had the highest Cars content. The increase of the carotenoid content in the double mutant could be a photoprotective response to compensate the presence of impaired regulations of photosynthesis. Nevertheless the $p p h 1 / n p q 4$ mutant is more susceptible to photoinhibition than $p p h 1$ and wild type plants and it is not more resistant to high light stress than the single $n p q 4$ mutant. This suggests that the increase of carotenoids is not sufficient to compensate photoprotection in the absence of an efficient NPQ. The reason for a higher content of carotenoids (on a Chls basis) in plants blocked in State II is not clear. This could be also related to a decrease in total Chls in these mutants, but this point will need further investigation. 


\section{Plant lacking PsbS and PPH1 shows enhanced growth and flower and siliques production in low light condition}

Plants growing in natural environment undergo multiple photosynthesis regulations to respond to different light intensities (often rapidly fluctuating) and finally optimise plant fitness and survival.

State transitions, as discussed previously, are an important regulation under non saturating light. Plants deficient in the phosphatase PPH1/TAP38 are blocked in State II, causing a constitutive increase of the antenna size of PSI as compared with wild type plants (Pribil et al. 2010; Shapiguzov et al. 2010). Interestingly, it has been reported that the pph1 mutant has enhanced photosynthetic performance in low light, as indicated by an increase in ФPSII, a decrease of 1-qP (reduction state of the primary electron acceptor in PSII), and finally a growth advantage under constant low-light intensity (Pribil et al. 2010). This has been explained by a more robust photosynthetic electron flow under conditions inducing State II in the pphl mutant as compared with wild type plants (Pribil et al. 2010).

Thermal dissipation of excess energy is a regulative process important under high light. Even if in competition with photosynthesis, NPQ does not reduce photochemistry at high irradiance, since light is in excess with respect to the photosynthetic capacity. On the contrary, under fluctuating irradiance, it is proposed that dissipation of energy could reduce the quantum yield of photosynthesis and thereby $\mathrm{CO}_{2}$ assimilation (Long et al. 1994; Hubbart et al. 2012; Kromdijk et al. 2016).

Since PsbS-dependent energy dissipation should not be necessary under controlled non-stressing conditions, we checked the effect of the lack of PsbS on growth under very low light conditions.

WT and mutant plants were grown 17 days in normal light (this is necessary to avoid excessive stem elongation of germinating plantlets) and then placed under stable low light conditions $\left(\sim 20 \mu \mathrm{mol}\right.$ photons $\left.\mathrm{m}^{-2} \mathrm{~s}^{-1}\right)$ in a growth chamber. Three experiences (test 1,2,3) were performed under the same illumination conditions. The dry weights of the rosettes have been determined after 20,33, 37 days of growth in low light for test 1,2,3, respectively. Results (Fig. 3) show a clear improvement of plant growth (dry weight) of all mutants as compared with wild type in low light. The absence of PsbS in the $n p q 4$ mutant led to a growth increase of $60 \%, 10 \%$ and $14 \%$, respectively in test 1 , 2,3 , while this increase was $28 \%, 19 \%, 9 \%$ in the absence of the PPH1 protein. Interestingly, in the double mutant the increase was even higher, being $63 \%, 25 \%$ and $26 \%$. All growth differences were statistically significant with respect to the wild type. The comparison of growth of double mutant versus single mutants showed differences statistically significant in some case (noted with \# in Fig. 3), with the double mutant showing the best growth. The general trend for the double mutant suggests that a cumulative effect on growth of the two mutations is present.

We observed that the growth increase under low light of mutants as compared with wild type plants was reduced with increasing times (Fig. 3). This could be explained by a reduction of growth due to the entering in the reproductive phase thus buffering the difference between wild type and mutant plants.

A similar result was found for the number of siliques and flowers (Fig. 4). We found an increase of siliques and flowers number in the $n p q 4$ mutant of $\sim 12 \%$ (with p-value $=7 \%$ ) for test 1 and $11 \%$ (with p-value $=0.2 \%$ ) for test 2. The increase of siliques and flowers number in the pph1/npq4 mutant with respect to the wild type was $\sim 23 \%$ with high confidence ( $\mathrm{p}$-value $<0.06 \%$ for both tests), while for the $p p h 1$ mutant this value was $23 \%$ ( $\mathrm{p}$-value $=1 \%$ ) for test 1 and $16 \%$ (p-value $=0.03 \%$ ) for test 2 . Siliques and flowers number increase in the pph1/npq4 mutant 
compared with single mutants was statistically significant in test 2 , suggesting that, as for growth, a cumulative effect of the two mutations might exist.

To test the effect of an over accumulation of PsbS, we also checked growth and silique production of L17 plants, a line obtained by transformation of WT (Col-0) plants with a genomic fragment containing the $p s b S$ gene and over accumulating PsbS (Li et al. 2002b). L17 plants showed a clear lower growth than $n p q 4$ plants, and similar or lower growth than WT plants (Fig. 5a). Silique production after 55 days in LL was the highest in $n p q 4$ plants and the lowest in L17 plants (Fig. 5b). During the same experiment, we also noted that after 49 days at LL, all ten $n p q 4$ plants, seven WT plants and only one L17 plant had at least one silique per plant.

Finally, we checked the PSII quantum yield under low and normal light of WT, npq4, pph1 and the double mutant plants (Table 2). We found a small but statistically significant difference between mutants and wild type for PSII yield at low light intensity, which could explain the improved growth of mutants, but we were not able to observe a higher PSII yield for the double mutant as compared with single mutants. This could be explained by technical issues to discriminate very small variations in fluorescence parameters. However, a little difference in PSII yield could be amplified when total growth is analysed after several days in low light. Indeed growth cumulates the effect of an improved photosynthesis and is a better indicator than the instantaneous yield of PSII to discriminate photochemical efficiencies of different genotypes during prolonged growth under low light. As alternative explanation, the increased growth in the pphl/npq4 double mutant is not only dependent on $\Phi$ PSII, but a positive effect from association of both mutations would lead to an improved growth that does not depend on the addition of ФPSII increases of single mutants (which we did not detect).

Under normal light, PSII yield (Table 2) and growth (Fig. 6) were similar in mutant and wild type plants, suggesting that the absence of both PsbS and PPH1 proteins does not cause a negative effect on plants in this condition.

Even if other investigations will be necessary to evaluate the phenotype of the pphl mutations (alone and in combination with the $n p q 4$ mutation) under other light conditions, as PSI light (light enriched in far red) and fluctuating light, our results support the fact that under constant high light, the pph1 mutation has little effect on photoprotective ability and at low light intensity the $p p h 1$ mutant shows an improved growth, as previously shown by Pribil and co-workers (Pribil et al. 2010), and also an increased flowers and siliques production (this work).

Interestingly, the lack of PsbS in the npq4 mutant leads to a similar enhanced growth and a higher number of flowers and siliques under low light conditions. A possible explanation for the enhanced growth of $n p q 4$ mutants is that a higher photosynthetic activity is due to the absence of PsbS, which could be partially active even at very low light and induce some grana membrane reorganisation favourable to energy dissipation (Horton et al. 2005; Kiss et al. 2008; Betterle et al. 2009; Kereiche et al. 2010). The fact that PsbS accumulation is significantly down-regulated in shade plants as Physcomitrella patens (Gerotto et al. 2011), as well as not or barely detectable in other green organisms living in shade conditions (Bonente et al. 2008), is in agreement with a possible negative effect of this protein under very limiting light conditions. The result on growth and silique production obtained with L17 plants, which over accumulate PsbS and show lower performances under LL, support a negative effect of PsbS under low light. It should be noted however the lower performances in transformed plants can be obtained for several reasons (such as mutation at the insertion site, ectopic protein accumulation) and, in the case of L17 plants, we cannot 
exclude pleitropic negative effects on photosynthesis due to an overaccumulation of PsbS in membrane. However, a positive effect on growth of mutant lines as compared to WT plants is generally much unexpected. This support the fact the improved performances of $n p q 4$ plants at low light are associated with the lack of PsbS. It is also interesting to observe that the effect of the $p p h 1$ and $n p q 4$ mutations seems to be cumulative under our controlled low light conditions. However in our work (Fig. 1 and 2) we did not detect interactions between the two photosynthesis regulations (PsbS-NPQ and PPH1-state transitions), which thus seem to work independently.

In conclusion, these results are in line with the idea that several photosynthetic regulations have evolved in plants to optimise fitness rather than net plant growth under natural conditions (Murchie and Niyogi 2011, Kromdijk et al. 2016). Therefore it might be possible to improve performances of plants grown under particular controlled condition by acting on photosynthesis at a molecular level. Similar mutations for NPQ and state transitions in agronomic relevant plants that experience light conditions strongly limiting for growth would be interesting to test to estimate the potential for applications of our results found on the model plant Arabidopsis.

\section{Acknowledgements}

This work was supported by the French National Research Agency Grant ANR-2012-JCJC-0001-01, the 322 project of the Vietnamese Government and the project of Vietnam University of Forestry, 2014. Bernard Genty (BIAM, Biosciences and Biotechnologies Institute of Aix-Marseille - AMU/CRNS/CEA) is thanked for helpful discussion.

\section{Author Contributions}

SC and TTHK designed the research; TTHK performed most of the experiments with some contribution by SC; SC and TTHK wrote the paper; CR provided general supervision, discussion and paper revision.

\section{Conflict of interest}

All authors declare that they have no conflict of interest. 


\section{References}

Ahn TK, Avenson TJ, Ballottari M, Cheng YC, Niyogi KK, Bassi R, Fleming GR (2008) Architecture of a chargetransfer state regulating light harvesting in a plant antenna protein. Science 320:794-797

Allen JF (1992) Protein phosphorylation in regulation of photosynthesis. Biochim Biophys Acta 1098:275-335

Allen JF (2003) Botany. State transitions--a question of balance. Science 299:1530-1532

Alonso JM, Stepanova AN, Leisse TJ, Kim CJ, Chen H, Shinn P, Stevenson DK, Zimmerman J, Barajas P, Cheuk R, Gadrinab C, Heller C, Jeske A, Koesema E, Meyers CC, Parker H, Prednis L, Ansari Y, Choy N, Deen H, Geralt M, Hazari N, Hom E, Karnes M, Mulholland C, Ndubaku R, Schmidt I, Guzman P, Aguilar-Henonin L, Schmid M, Weigel D, Carter DE, Marchand T, Risseeuw E, Brogden D, Zeko A, Crosby WL, Berry CC, Ecker JR (2003) Genome-wide insertional mutagenesis of Arabidopsis thaliana. Science 301:653-657

Asada K (2002) THE WATER-WATER CYCLE IN CHLOROPLASTS: Scavenging of Active Oxygens and Dissipation of Excess Photons. Annu Rev Plant Physiol Plant Mol Biol 50:601-639

Awan SF, Khan IA, Khan AA, Schneider A, Altaf A, Ahmad R, Leister D (2008) Screening of Arabidopsis mutant for functional genomic studies. Pak J Bot 40:2071-2080

Bellafiore S, Barneche F, Peltier G, Rochaix JD (2005) State transitions and light adaptation require chloroplast thylakoid protein kinase STN7. Nature 433:892-895

Betterle N, Ballottari M, Zorzan S, de Bianchi S, Cazzaniga S, Dall'osto L, Morosinotto T, Bassi R (2009) Lightinduced dissociation of an antenna hetero-oligomer is needed for non-photochemical quenching induction. $\mathrm{J}$ Biol Chem 284:15255-15266

Bonaventura C, Myers J (1969) Fluorescence and oxygen evolution from Chlorella pyrenoidosa. Biochim Biophys Acta 189:366-383

Bonente G, Ballottari M, Truong TB, Morosinotto T, Ahn TK, Fleming GR, Niyogi KK, Bassi R (2011) Analysis of LhcSR3, a protein essential for feedback de-excitation in the green alga Chlamydomonas reinhardtii. PLoS Biol 9:e1000577

Bonente G, Howes BD, Caffarri S, Smulevich G, Bassi R (2008) Interactions between the photosystem II subunit PsbS and xanthophylls studied in vivo and in vitro. J Biol Chem 283:8434-8445

Campoli C, Caffarri S, Svensson JT, Bassi R, Stanca AM, Cattivelli L, Crosatti C (2009) Parallel pigment and transcriptomic analysis of four barley albina and xantha mutants reveals the complex network of the chloroplastdependent metabolism. Plant Mol Biol 71:173-191

Crepin A, Caffarri S (2015) The specific localizations of phosphorylated Lhcb1 and Lhcb2 isoforms reveal the role of Lhcb2 in the formation of the PSI-LHCII supercomplex in Arabidopsis during state transitions. Biochim Biophys Acta Bioenerg 1847:1539-1548

Croce R, Canino g, Ros F, Bassi R (2002) Chromophore organization in the higher-plant photosystem II antenna protein CP26. Biochemistry 41:7334-7343

Demmig-Adams B (1990) Carotenoids and photoprotection in plants: A role for the xanthophyll zeaxanthin. Biochim Biophys Acta 1020:1-24

Depege N, Bellafiore S, Rochaix JD (2003) Role of chloroplast protein kinase Stt7 in LHCII phosphorylation and state transition in Chlamydomonas. Science 299:1572-1575

Dominici P, Caffarri S, Armenante F, Ceoldo S, Crimi M, Bassi R (2002) Biochemical properties of the PsbS subunit of photosystem II either purified from chloroplast or recombinant. J Biol Chem 277:22750-22758

Dong L, Tu W, Liu K, Sun R, Liu C, Wang K, Yang C (2015) The PsbS protein plays important roles in photosystem II supercomplex remodeling under elevated light conditions. J Plant Physiol 172:33-41

Funk C, Adamska I, Green BR, Andersson B, Renger G (1995) The nuclear-encoded chlorophyll-binding photosystem II-S protein is stable in the absence of pigments. J Biol Chem 270:30141-30147

Galka P, Santabarbara S, Khuong TTH, Degand H, Morsomme P, Jennings RC, Boekema EJ, Caffarri S (2012) Functional analyses of the plant photosystem I-light-harvesting complex II supercomplex reveal that lightharvesting complex II loosely bound to photosystem II is a very efficient antenna for photosystem I in state II. Plant Cell 24:2963-2978

Genty B, Briantais JM, Baker NR (1989) The relationship between the quantum yield of photosynthetic electron transport and quenching of chlorophyll fluorescence. Biochim Biophys Acta 990:87-92

Gerotto C, Alboresi A, Giacometti GM, Bassi R , Morosinotto T (2011) Role of PSBS and LHCSR in Physcomitrella patens acclimation to high light and low temperature. Plant Cell Environ 34:922-932

Gerotto C, Alboresi A, Meneghesso A, Jokel M, Suorsa M, Aro EM, Morosinotto T (2016) Flavodiiron proteins act as safety valve for electrons in Physcomitrella patens. Proc Natl Acad Sci USA 113, 12322-12327

Gilmore AM, Yamamoto HY (1992) Dark induction of zeaxanthin-dependent nonphotochemical fluorescence quenching mediated by ATP. Proc Natl Acad Sci USA 89:1899-1903 
Golan T, Muller-Moule P, Niyogi KK (2006) Photoprotection mutants of Arabidopsis thaliana acclimate to high light by increasing photosynthesis and specific antioxidants. Plant Cell Environ 29:879-887

Gorbunov MY, Kuzminov FI, Fadeev VV, Kim JD, Falkowski PG (2011) A kinetic model of non-photochemical quenching in cyanobacteria. Biochim Biophys Acta Bioenerg 1807:1591-1599

Głowacka K, Kromdijk J, Kucera K, Xie J, Cavanagh AP, Leonelli L, Leakey ADB, Ort DR, Niyogi KK, Long SP (2018) Photosystem II Subunit S overexpression increases the efficiency of water use in a field-grown crop. Nature Communications 9:868. doi:10.1038/s41467-018-03231-x

Haldrup A, Jensen PE, Lunde C, Scheller HV (2001) Balance of power: a view of the mechanism of photosynthetic state transitions. Trends Plant Sci 6:301-305

Havaux M, Niyogi KK (1999) The violaxanthin cycle protects plants from photooxidative damage by more than one mechanism. Proc Natl Acad Sci USA 96:8762-8767

Holzwarth AR, Miloslavina Y, Nilkensb M, Jahnsb P (2009) Identification of two quenching sites active in the regulation of photosynthetic light-harvesting studied by time-resolved fluorescence. Chem Phys Lett 483 262-267

Horton P, Ruban AV (2005) Molecular design of the photosystem II light-harvesting antenna: photosynthesis and photoprotection. J Exp Bot 56:365-373

Horton P, Wentworth M, Ruban A (2005) Control of the light harvesting function of chloroplast membranes: The LHCII-aggregation model for non-photochemical quenching. FEBS Lett 579:4201-4206

Hubbart S, Ajigboye OO, Horton P, Murchie EH (2012) The photoprotective protein PsbS exerts control over CO(2) assimilation rate in fluctuating light in rice. Plant J 71:402-412

Johnson MP, Ruban AV (2010) Arabidopsis plants lacking PsbS protein possess photoprotective energy dissipation. Plant J 61:283-289

Johnson MP, Ruban AV (2011) Restoration of rapidly reversible photoprotective energy dissipation in the absence of PsbS protein by enhanced DeltapH. J Biol Chem 286:19973-19981

Kalituho L, Grasses T, Graf M, Rech J, Jahns P (2006) Characterization of a nonphotochemical quenching-deficient Arabidopsis mutant possessing an intact PsbS protein, xanthophyll cycle and lumen acidification. Planta 223:532541

Kasajima I, Ebana K, Yamamoto T, Takahara K, Yano M, Kawai-Yamada M, Uchimiya H (2011) Molecular distinction in genetic regulation of nonphotochemical quenching in rice. Proc Natl Acad Sci USA 108:1383513840

Kereiche S, Kiss AZ, Kouril R, Boekema EJ, Horton P (2010) The PsbS protein controls the macro-organisation of photosystem II complexes in the grana membranes of higher plant chloroplasts. FEBS Lett 584:759-764

Kiss AZ, Ruban AV, Horton P (2008) The PsbS protein controls the organization of the photosystem II antenna in higher plant thylakoid membranes. J Biol Chem 283:3972-3978

Krah NM, Logan BA (2010) Loss of psbS expression reduces vegetative growth, reproductive output, and lightlimited, but not light-saturated, photosynthesis in Arabidopsis thaliana (Brassicaceae) grown in temperate light environments. Am J Bot 97:644-649

Krieger-Liszkay A (2005) Singlet oxygen production in photosynthesis. J Exp Bot 56:337-346

Kromdijk J, Glowacka K, Leonelli L, Gabilly ST, Iwai M, Niyogi KK, Long SP (2016) Improving photosynthesis and crop productivity by accelerating recovery from photoprotection. Science 354:857-861

Kulheim C, Agren J, Jansson S (2002) Rapid regulation of light harvesting and plant fitness in the field. Science 297:91-93

Lemeille S, Willig A, Depege-Fargeix N, Delessert C, Bassi R, Rochaix JD (2009) Analysis of the chloroplast protein kinase Stt7 during state transitions. PLoS Biol 7:e45

Li X, Phippard A, Pasari J, Niyogi K (2002a) Structural-functional analysis of Photosystem II subunit S (PsbS) in vivo. Funct Plant Biol 29:1131-1139

Li XP, Bjorkman O, Shih C, Grossman AR, Rosenquist M, Jansson S, Niyogi KK (2000) A pigment-binding protein essential for regulation of photosynthetic light harvesting. Nature 403:391-395

Li XP, Gilmore AM, Caffarri S, Bassi R, Golan T, Kramer D, Niyogi KK (2004) Regulation of photosynthetic light harvesting involves intrathylakoid lumen $\mathrm{pH}$ sensing by the PsbS protein. J Biol Chem 279:22866-22874

Li XP, Muller-Moule P, Gilmore AM, Niyogi KK (2002b) PsbS-dependent enhancement of feedback de-excitation protects photosystem II from photoinhibition. Proc Natl Acad Sci U S A 99:15222-15227

Li Z, Wakao S, Fischer BB, Niyogi KK (2009) Sensing and responding to excess light. Annu Rev Plant Biol 60:239-260

Long SP, Humphries S, Falkowski PG (1994) Photoinhibition of photosynthesis in nature. Annu Rev Plant Physiol Plant Mol Biol 45:633-662 
Mao HB, Li GF, Ruan X, Wu QY, Gong YD, Zhang XF, Zhao NM (2002) The redox state of plastoquinone pool regulates state transitions via cytochrome b6f complex in Synechocystis sp. PCC 6803. FEBS Lett 519:82-86

Mekala NR, Suorsa M, Rantala M, Tikkanen M, Aro EM (2015) Plants Actively Avoid State Transitions upon Changes in Light Intensity: Role of Light-Harvesting Complex II Protein Dephosphorylation in High Light. Plant Physiol 168:721-734

Melis A (1999) Photosystem-II damage and repair cycle in chloroplasts: what modulates the rate of photodamage ? Trends Plant Sci 4:130-135

Minagawa J (2011) State transitions--the molecular remodeling of photosynthetic supercomplexes that controls energy flow in the chloroplast. Biochim Biophys Acta Bioenerg 1807:897-905

Muller P, Li XP, Niyogi KK (2001) Non-photochemical quenching. A response to excess light energy. Plant Physiol 125:1558-1566

Munekage Y, Hashimoto M, Miyake C, Tomizawa KI, Endo T, Tasaka M, Shikanai T (2004) Cyclic electron flow around photosystem I is essential for photosynthesis. Nature 429:579-582

Murata N (1969) Control of excitation transfer in photosynthesis. I. Light-induced change of chlorophyll a fluorescence in Porphyridium cruentum. Biochim Biophys Acta 172:242-251

Murchie EH, Niyogi KK (2011) Manipulation of photoprotection to improve plant photosynthesis. Plant Physiol 155:86-92

Niyogi K, Truong T (2013) Evolution of flexible non-photochemical quenching mechanisms that regulate light harvesting in oxygenic photosynthesis. Curr Opin Plant Biol 16 307-314

Niyogi KK, Grossman AR, Bjorkman O (1998) Arabidopsis mutants define a central role for the xanthophyll cycle in the regulation of photosynthetic energy conversion. Plant Cell 10:1121-1134

Niyogi KK, Li XP, Rosenberg V, Jung HS (2005) Is PsbS the site of non-photochemical quenching in photosynthesis? J Exp Bot 56:375-382

Pribil M, Pesaresi P, Hertle A, Barbato R, Leister D (2010) Role of Plastid Protein Phosphatase TAP38 in LHCII Dephosphorylation and Thylakoid Electron Flow. Plos Biology 8:e1000288

Puthiyaveetil S, Ibrahim IM, Allen JF (2012) Oxidation-reduction signalling components in regulatory pathways of state transitions and photosystem stoichiometry adjustment in chloroplasts. Plant Cell Environ 35:347-359

Rintamaki E, Martinsuo P, Pursiheimo S, Aro EM (2000) Cooperative regulation of light-harvesting complex II phosphorylation via the plastoquinol and ferredoxin-thioredoxin system in chloroplasts. Proc Natl Acad Sci USA 97:11644-11649

Rintamaki E, Salonen M, Suoranta UM, Carlberg I, Andersson B, Aro EM (1997) Phosphorylation of lightharvesting complex II and photosystem II core proteins shows different irradiance-dependent regulation in vivo. Application of phosphothreonine antibodies to analysis of thylakoid phosphoproteins. J Biol Chem 272:3047630482

Rochaix JD (2014) Regulation and dynamics of the light-harvesting system. . Annu Rev Plant Biol 65:287-309

Ruban AV (2017) Quantifying the efficiency of photoprotection. Philos Trans R Soc Lond B Biol Sci 372

Ruban AV, Berera R, Ilioaia C, van Stokkum IHM, Kennis JTM, Pascal AA, van Amerongen H, Robert B, Horton P, van Grondelle R (2007) Identification of a mechanism of photoprotective energy dissipation in higher plants. Nature 450:575-578

Ruban AV, Johnson MP, Duffy CD (2012) The photoprotective molecular switch in the photosystem II antenna. Biochim Biophys Acta Bioenerg 1817:167-181

Ruban AV, Murchie EH (2012) Assessing the photoprotective effectiveness of non-photochemical chlorophyll fluorescence quenching: A new approach. Biochim Biophys Acta Bioenerg 1817:977-982

Sacharz J, Giovagnetti V, Ungerer P, Mastroianni GV, Ruban AV (2017) The xanthophyll cycle affects reversible interactions between PsbS and light-harvesting complex II to control non-photochemical quenching. Nature Plants 3. doi:doi:10.1038/nplants.2016.225

Shapiguzov A, Ingelsson B, Samol I, Andres C, Kessler F, Rochaix JD, Vener AV, Goldschmidt-Clermont M (2010) The PPH1 phosphatase is specifically involved in LHCII dephosphorylation and state transitions in Arabidopsis. Proc Natl Acad Sci USA 107:4782-4787

Shikanai T, Yamamoto H (2017) Contribution of Cyclic and Pseudo-cyclic Electron Transport to the Formation of Proton Motive Force in Chloroplasts. Mol Plant 10:20-29

Sonoike K (2011) Photoinhibition of photosystem I. Physiol Plant 142:56-64

Sonoike K, Terashima I, Iwaki M, Itoh S (1995) Destruction of photosystem I iron-sulfur centers in leaves of Cucumis sativus L. by weak illumination at chilling temperatures. FEBS Lett 362:235-238 
Suorsa M, Grieco M, Jarvi S, Tikkanen M, Jansson S, Aro EM, Pietrzykowska M, Paakkarinen V, Nurmi M, Rantala M, Kangasjarvi S (2012) PROTON GRADIENT REGULATION5 Is Essential for Proper Acclimation of Arabidopsis Photosystem I to Naturally and Artificially Fluctuating Light Conditions. Plant Cell 24:2934-2948

Sylak-Glassman EJ, Malnoe A, De Re E, Brooks MD, Fischer AL, Niyogi KK, Fleming GR (2014) Distinct roles of the photosystem II protein PsbS and zeaxanthin in the regulation of light harvesting in plants revealed by fluorescence lifetime snapshots. Proc Natl Acad Sci USA 111:17498-17503

Tibiletti T, Auroy P, Peltier G, Caffarri S (2016) Chlamydomonas reinhardtii PsbS Protein Is Functional and Accumulates Rapidly and Transiently under High Light. Plant Physiol 171:2717-2730

Tikkanen M, Grieco M, Kangasjarvi S, Aro EM (2010) Thylakoid protein phosphorylation in higher plant chloroplasts optimizes electron transfer under fluctuating light. Plant Physiol 152:723-735

Tikkanen M, Nurmi M, Kangasjarvi S, Aro EM (2008) Core protein phosphorylation facilitates the repair of photodamaged photosystem II at high light. Biochim Biophys Acta Bioenerg 1777:1432-1437

Tjus SE, Møller BL, Scheller HV (1998) Photosystem I is an early target of photoinhibition in barley illuminated at chilling temperatures. Plant Physiol 116:755-764

Veeranjaneyulu K, Leblanc RM (1994) Action Spectra of Photosystems I and II in State 1 and State 2 in Intact Sugar Maple Leaves. Plant Physiol 104:1209-1214

Wilhelm C, Selmar D (2011) Energy dissipation is an essential mechanism to sustain the viability of plants: The physiological limits of improved photosynthesis. J Plant Physiol 168:79-87

Wollman FA (2001) State transitions reveal the dynamics and flexibility of the photosynthetic apparatus. Embo J 20:3623-3630

Zhang S, Scheller HV (2004) Photoinhibition of photosystem I at chilling temperature and subsequent recovery in Arabidopsis thaliana. Plant Cell Physiol 45:1595-1602 


\section{FIGURES and TABLES}

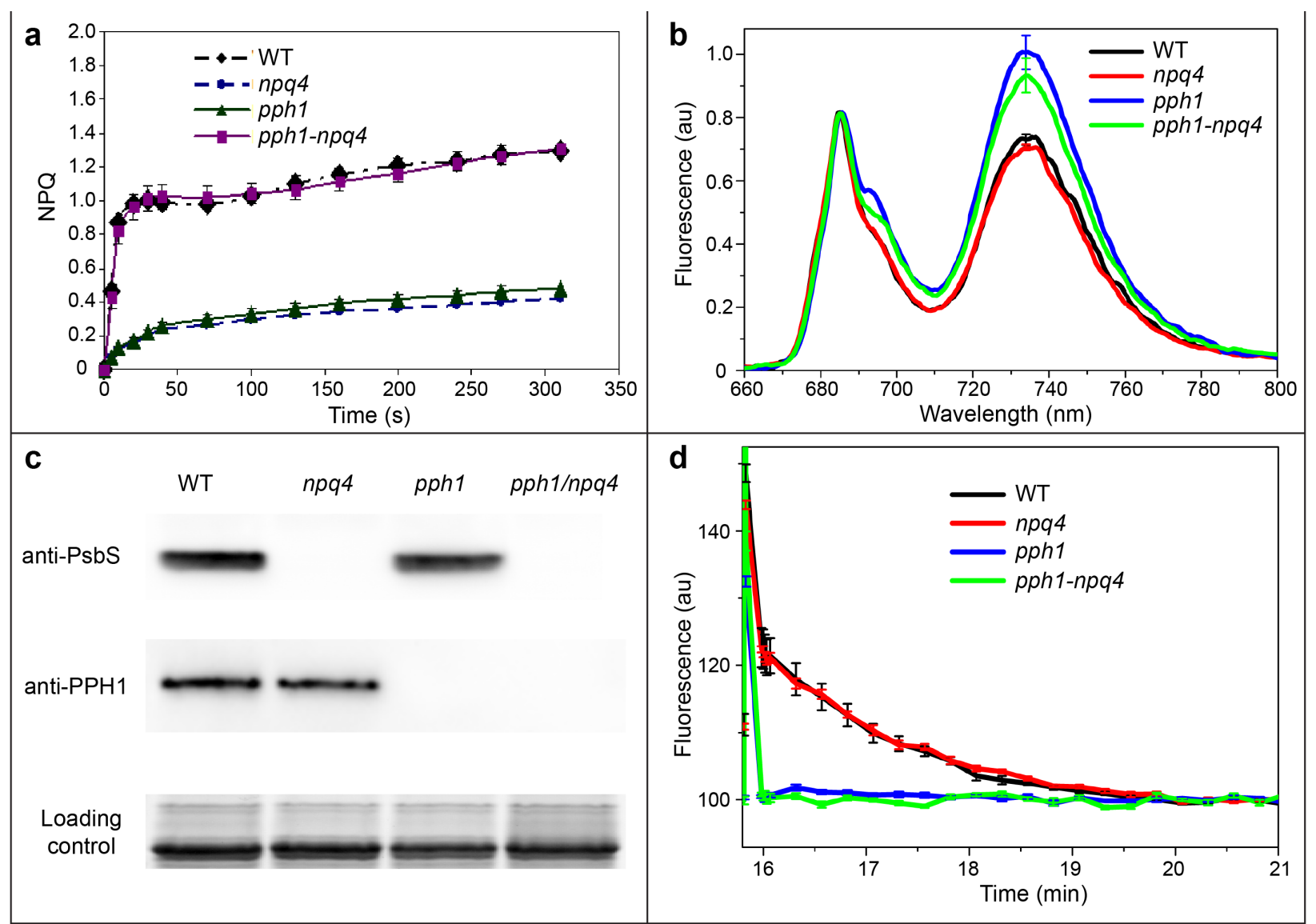

Figure 1. a NPQ induced by actinic light at $1200 \mu \mathrm{mol} \mathrm{m} \mathrm{m}^{-2}$ on plants grown 35 days in NL measured with a PAM fluorometer. The curves represent the average values and their standard error (SE) of 6 measurements on distinct plants for each genotype. b 77K fluorescence emission spectra of leaves from wild type (WT) and mutants plants. Preliminarily, plants have been exposed for $45 \mathrm{~min}$ to a far-red light to induce State I. The spectra were normalized at the maximum PSII emission (peak at $684 \mathrm{~nm}$ ). Traces are the average of replicates on 4 independent plants for each genotype. SE at the maximum emission of PSI is indicated. c Immunoblot analysis to test the absence of PsbS and PPH1 proteins in the double pph1/npq4 mutant. Specific antibodies against PsbS and PPH1 have been used on thylakoid proteins purified from a pool of several plants for each genotype (grown in NL). Proteins were loaded on an SDS-PAGE on a chlorophyll basis $(1.5 \mu \mathrm{g}$ of total Chls). The loading control is a Sypro stained gel (Lhe region $\sim 22-28 \mathrm{kDa}$ ) run with the same sample preparations and in the same 10-well gel. d Kinetics of State I to State II transition induced by blue light after $15 \mathrm{~min}$ of State I induction by far red light detected as fluorescence decrease (methods described in Crepin et al. 2015). Average curves of 8 plants for each genotype and SE are shown. For all experiments here shown, F3 plants have been used for the double mutants. Homozygosis was further checked by decreased NPQ induction and lack of state transitions using an imaging PAM fluorometer (supplemental Fig. S2). 


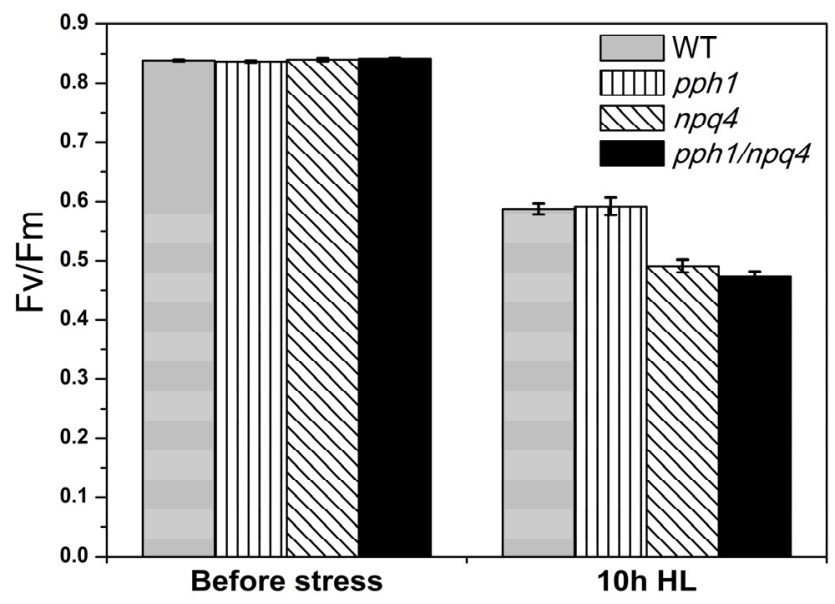

Figure 2. Maximum quantum yield of PSII ( $\mathrm{Fv} / \mathrm{Fm})$ measured before and after treatment by high light. Plants grown $\sim 3$ weeks in NL were treated under $1500 \mu \mathrm{mol} \mathrm{m}^{-2} \mathrm{~s}^{-1}$ and $12^{\circ} \mathrm{C}$ in a cold room for $10 \mathrm{hr}$. Fv/Fm values ( $\pm \mathrm{SE}$ ) are the average values of measurements using an imaging PAM system on 10 plants for genotype. The full plant was imaged (see Fig. S3 for an example). The Fv/Fm decrease of $n p q 4$ and $p p h 1 / n p q 4$ compared with wild type is statistically significant with p-value $<0.001$. Difference between WT and $p p h 1$ and between $n p q 1$ and $p p h 1 / n p q 4$ were not statistically significant. A high light stress experiment at normal temperature is presented in supplemental Fig. S4 and provided similar conclusions (that is comparable Fv/Fm decrease in WT and pphl plants and more pronounced and comparable decrease in $n p q 4$ and $p p h 1 / n p q 4$ plants).

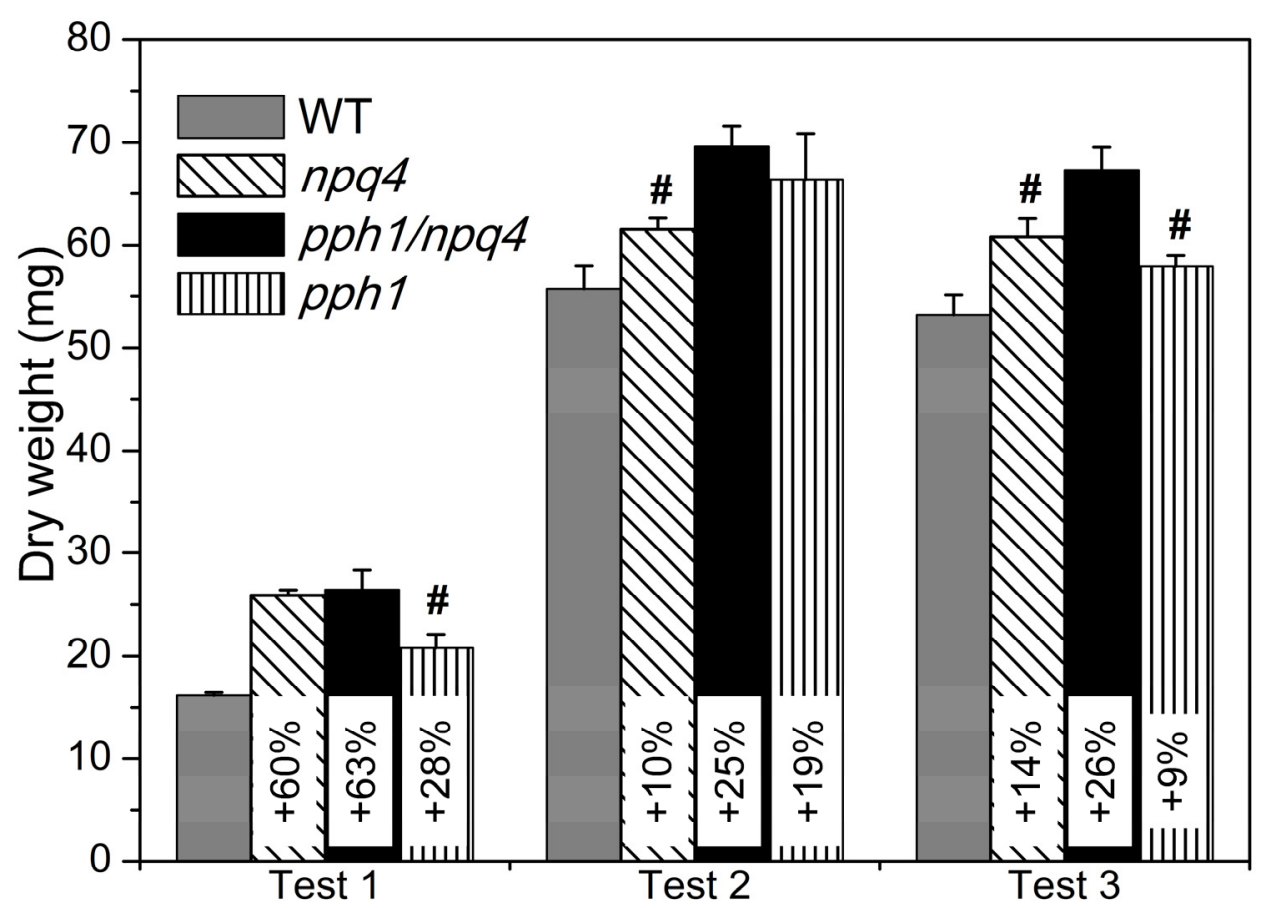

Figure 3. Growth of wild type, double and single mutants were determined as dry weight of plants grown for 17 days in NL then 20,33, 37 days in low light for test 1, test 2 and test 3, respectively. The figure present the average values and SE of 6, 10, 12 plants for each genotype for test 1 , test 2 and test 3, respectively. Growth differences between wild type and mutants are all statistically significant with $\mathrm{p}$-value $<5 \%$. Statistically significant differences between the pphl/npq4 double mutant and the single mutants are indicated with the \# symbol above the single mutant. The relative growth increase of mutant plants in comparison with wild type plants is indicated in the bar inset. 


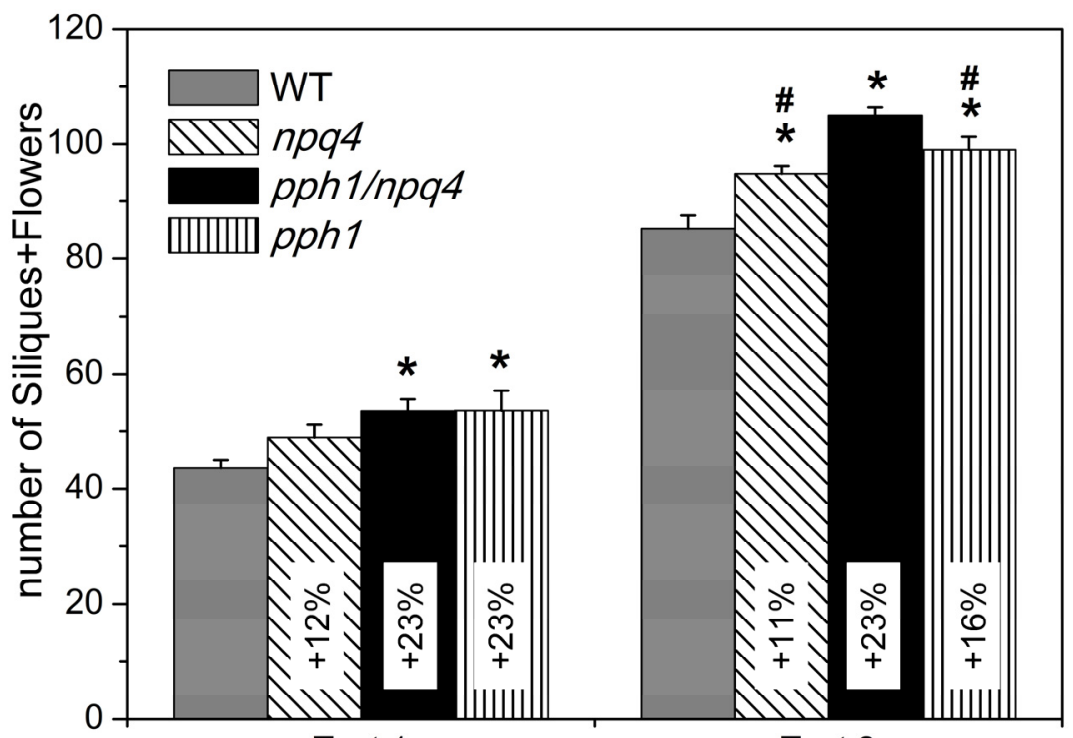

Test 1

Test 2

Figure 4. Number of siliques + flowers of wild type and mutant plants in low light condition was measured on plants grown 17 days in NL and then 24 days in LL (Test 1) or 37 days in LL (Test 2). The figure shows the average values and SE from 12 plants for each genotype. Statistically significant differences between mutants and wild type are indicated ( $\mathrm{p}$-value $<5 \%$; indicated with the ${ }^{*}$ symbol). In test 2 , the silique + flower number increase in the pph1/npq4 double mutant is statistically significant as compared with single mutants values ( $\mathrm{p}$-value $<5 \%$; indicated with the \# symbol). The relative increase of siliques+flowers number in mutant plants in comparison with wild type plants is indicated in the bar inset.

a

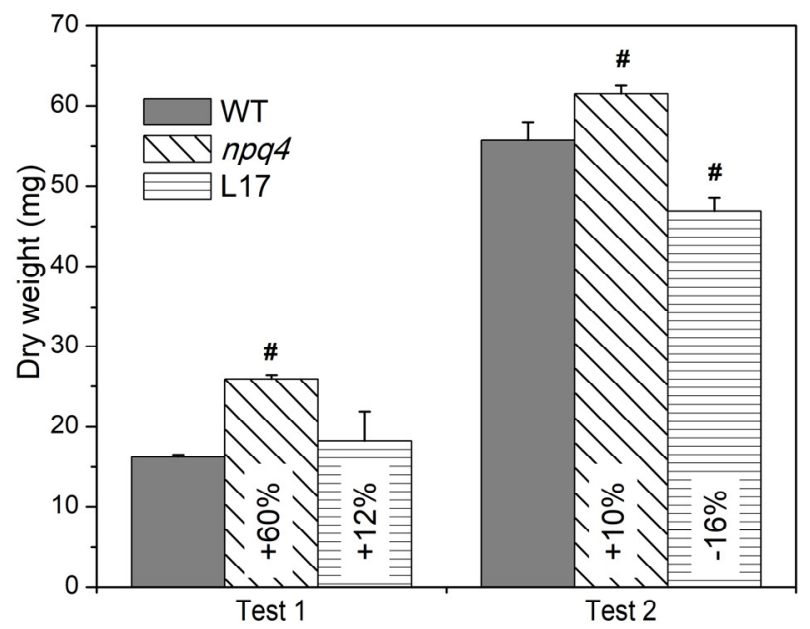

b

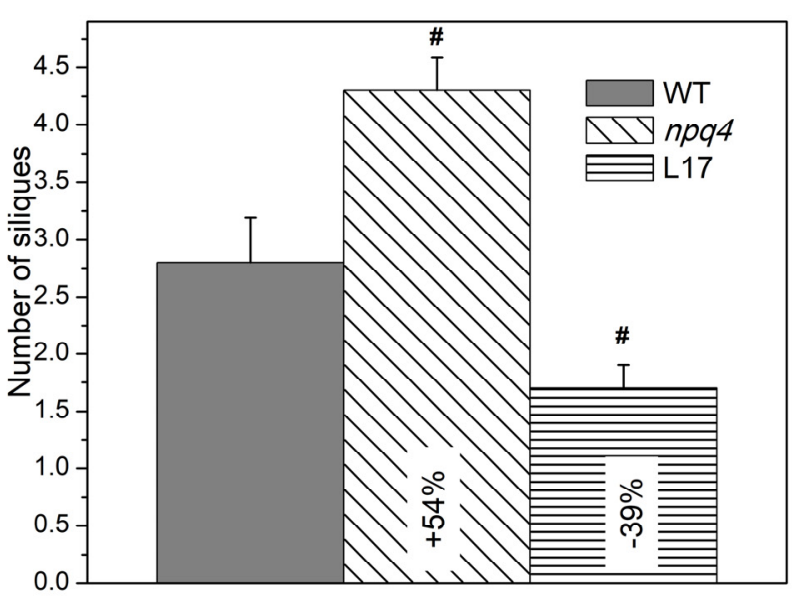

Figure 5. a Growth of wild type, npq4 and L17 (a line overexpressing PsbS) plants determined as dry weight of plants grown for 17 days in NL then 20 and 33 for test 1 and test 2, respectively (same test as in Fig. 3). The average values and SE of 6 and 10 plants for each genotype for test 1 and test 2, respectively, are shown. Statistically significant differences ( $\mathrm{p}$-value $<5 \%$ ) between wild type and mutants are indicated with the \# symbol. The relative growth increase or decrease in comparison with wild type plants is indicated in the bar inset. b Numbers of siliques per plant for WT, $n p q 4$ and L17 plants grown 55 days in LL. The average values and SE of 10 plants for each genotype are shown, and differences with respect to the WT are statistically significant (p-value $<5 \%$ ). 


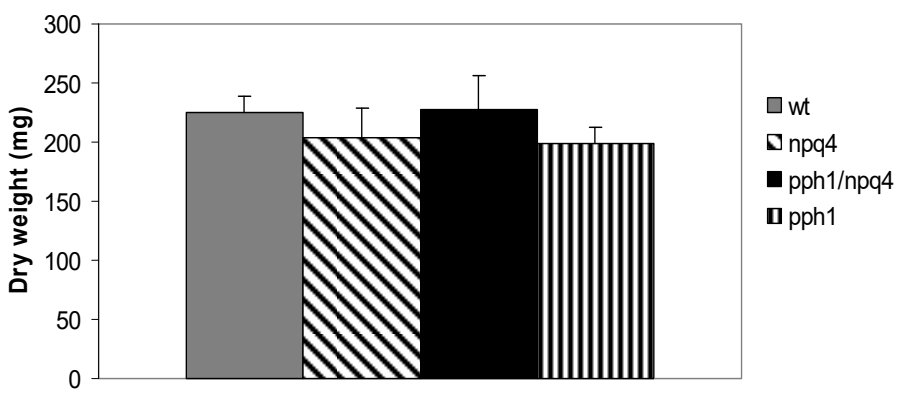

Figure 6. Dry weights of double mutant, single mutants and wild type plants grown 38 days in normal light. The figure shows average values and SE of 5 plants for each genotype. Differences between genotypes are not statistically significant ( $\mathrm{p}$-value $>38 \%$ for all test). 
Table 1. Leaf pigment composition of wild-type, $n p q 4, p p h 1$ and $p p h 1 / n p q 4$ mutants. The data are normalized to 100 total Chls. Neo, neoxanthin; Vio, violaxanthin; Ant, antheraxanthin; Lut, lutein, Zea, zeaxanthin, $\beta$-Car, $\beta$ carotene, VAZ, Vio+Ant+Zea. Analyses were performed on a pool of leaves from 15 plants for each genotype (3 replicates). Growth conditions: "LL", 18 days in normal light (NL) then 35 days in low light; "HL", 51 days in NL then 4.5 days $(16 \mathrm{D} / 8 \mathrm{~L})$ at high light. Light regimes: $\mathrm{NL}, 120 \mu \mathrm{mol} \mathrm{m}^{-2} \mathrm{~s}^{-1}$; LL, $20 \mu \mathrm{mol} \mathrm{m}^{-2} \mathrm{~s}^{-1}$; HL, $1600 \mu \mathrm{mol} \mathrm{m}^{-2} \mathrm{~s}^{-}$ ${ }^{1}$. The de-epoxidation index is indicated in the last column as ratio between $\left(0.5^{*} \mathrm{Ant}+\mathrm{Zea}\right) /(\mathrm{Vio}+\mathrm{Ant}+\mathrm{Zea})$. Ant at the numerator is multiplied by 0.5 since Ant has just one de-epoxidised ring, while Zea has both terminal rings deepoxidised. For all pigments and ratios, relative errors are lower than $5 \%$ (generally $\sim 1 \%$ ). Statistically significant differences (p-value $<5 \%$ ) are indicated in parenthesis: "w", "n", "p" and "pn" mean that a value is statistically different from the value in the same condition of the WT, npq4, pph1 and $p p h 1 / n p q 4$, respectively.

\begin{tabular}{|c|c|c|c|c|c|c|c|c|c|c|c|c|c|}
\hline Genotype & Light & $\mathrm{Chl}$ a/b & $\mathrm{NeO}$ & Vio & Ant & Lut & Zea & Chl b & Chl a & $\beta$ car & Chls/Cars & VAZ & $\begin{array}{c}\begin{array}{c}\text { De-epox } \\
\text { index }\end{array} \\
\end{array}$ \\
\hline \multirow{3}{*}{ WT } & LL & 2.98 & 3.84 & 2.65 & 0.00 & 13.20 & 0.00 & 25.14 & 74.86 & 7.17 & 3.72 & 2.65 & $0.0 \%$ \\
\hline & NL & $3.04(p)$ & $3.95(\mathrm{pn})$ & 3.04 & 0.00 & $13.63(n, p n)$ & 0.00 & $24.78(p)$ & $75.22(p)$ & $7.54(p)$ & $3.55(n, p n)$ & 3.04 & $0.0 \%$ \\
\hline & HL & $3.11(p)$ & $4.63(\mathrm{pn})$ & $4.13(p n, p)$ & $1.38(\mathrm{pn}, \mathrm{p})$ & $16.09(p n, p)$ & $0.9(p n, p)$ & $24.35(p)$ & $75.65(p)$ & $7.78(\mathrm{pn}, \mathrm{p})$ & $2.87(p n, p)$ & $6.4(p n, p)$ & $24.8 \%$ \\
\hline \multirow{3}{*}{ npq4 } & LL & 2.97 & 3.62 & 2.69 & 0.00 & 12.58 & 0.00 & 25.17 & 74.83 & 7.10 & 3.85 & 2.69 & $0.0 \%$ \\
\hline & NL & $2.98(p)$ & 3.46 & $2.49(p)$ & 0.00 & $12.7(w)$ & 0.00 & $25.10(p)$ & $74.90(p)$ & $7.14(p)$ & $3.88(w, p)$ & $2.49(p)$ & $0.0 \%$ \\
\hline & $\mathrm{HL}$ & $3.17(p)$ & $4.51(\mathrm{pn})$ & $4.08(p n, p)$ & $1.39(p n, p)$ & $16.93(p n, p)$ & $0.9(p n, p)$ & $23.97(p)$ & $76.03(p)$ & $8.49(\mathrm{pn}, \mathrm{p})$ & $2.75(\mathrm{pn}, \mathrm{p})$ & $6.38(p n, p)$ & $25.0 \%$ \\
\hline \multirow{3}{*}{$p p h 1 / n p q 4$} & LL & 2.99 & 3.50 & 2.55 & 0.00 & 13.48 & 0.00 & 25.08 & 74.92 & 7.16 & 3.75 & 2.55 & $0.0 \%$ \\
\hline & $\mathrm{NL}$ & 3.09 & $3.48(w)$ & $2.56(p)$ & 0.00 & $12.68(w)$ & 0.00 & 24.45 & 75.55 & $7.28(p)$ & $3.85(w, p)$ & $2.56(p)$ & $0.0 \%$ \\
\hline & HL & $3.15(p)$ & $5.90(w, n)$ & $6.57(w, n)$ & $1.73(w, n)$ & $22.86(w, n, p)$ & $1.62(w, n)$ & $24.08(p)$ & $75.92(p)$ & $10.98(w, n)$ & $2.01(w, n, p)$ & $9.92(w, n)$ & $25.1 \%$ \\
\hline \multirow{3}{*}{ pph1 } & LL & 3.08 & 3.62 & 2.79 & 0.00 & 12.68 & 0.00 & 24.50 & 75.50 & 7.28 & 3.79 & 2.79 & $0.0 \%$ \\
\hline & NL & $3.10(w, n)$ & 3.65 & $2.88(n, p n)$ & 0.00 & 12.91 & 0.00 & $24.38(w, n)$ & $75.62(w, n)$ & $7.99(w, n, p n)$ & $3.65(\mathrm{n}, \mathrm{pn})$ & $2.88(n, p n)$ & $0.0 \%$ \\
\hline & HL & $3.51(w, n, p n)$ & 5.30 & $5.64(w, n)$ & $1.67(w, n)$ & $19.69(w, n, p n)$ & $1.33(w, n)$ & $22.19(w, n, p n)$ & $77.81(w, n, p n)$ & $11.21(w, n)$ & $2.23(w, n, p n)$ & $8.64(w, n)$ & $25.1 \%$ \\
\hline
\end{tabular}

Table 2. PSII quantum yield (ФPSII) of plants in low light (LL; plants grown 20 days in NL and then 32 days in LL) and normal light (NL; plants grown 52 days in NL). Average values and SE of measurements on 8 plants per genotype, each plant measured on three leaves. The increase of PSII yield in mutants is statically significant compared to that of wild type in LL ( $\mathrm{p}$-value $<5 \%$ ), but the difference between double mutant and single mutants is not statistically significant. In NL, differences are not statistically significant.

\begin{tabular}{|c|c|c|c|c|}
\hline Light & WT & npq4 & pph1/npq4 & pph1 \\
\hline LL & $0.758 \pm 0.001$ & $0.771 \pm 0.001$ & $0.770 \pm 0.000$ & $0.772 \pm 0.001$ \\
\hline NL & $0.757 \pm 0.002$ & $0.759 \pm 0.003$ & $0.760 \pm 0.003$ & $0.762 \pm 0.004$ \\
\hline
\end{tabular}




\section{SUPPLEMENTAL INFORMATION}

\section{Photoprotection and growth under different lights of Arabidopsis single and double mutants for energy dissipation (npq4) and state transitions (pph1)}

Thi Thu Hương Khuong ${ }^{1,2,3}$, Christophe Robaglia ${ }^{1}$, Stefano Caffarri ${ }^{1}$

${ }^{1}$ Aix Marseille Université, CEA, CNRS, Biosciences and Biotechnologies Institute of Aix-Marseille (BIAM), Equipe de Luminy de Génétique et Biophysique des Plantes, 13009 Marseille, France.

${ }^{2}$ Cell Technology Laboratory - CFB - Vietnam National University of Forestry, Hanoi, Vietnam.

${ }^{3}$ The Key Laboratory of Enzyme and Protein Technology (KLEPT), Hanoi University of Science (HUS), Vietnam National University (VNU), Hanoi, Vietnam.

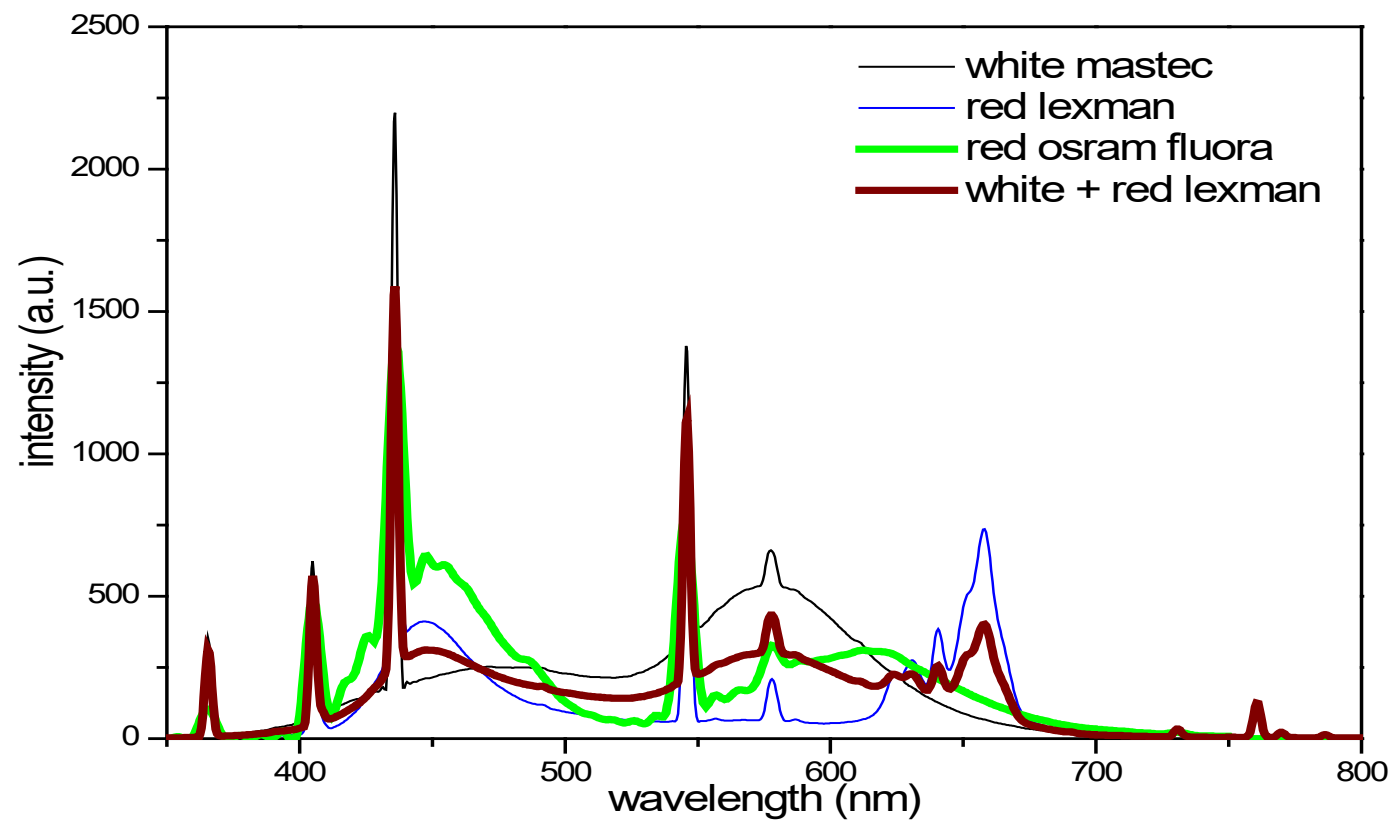

Figure S1. Light spectra of fluorescent lamps used for growth tests in low light. We used a mix of white (Mastec) and red (Lexman) lamps for test 1 and only Fluora lamps for test 2 and 3. 


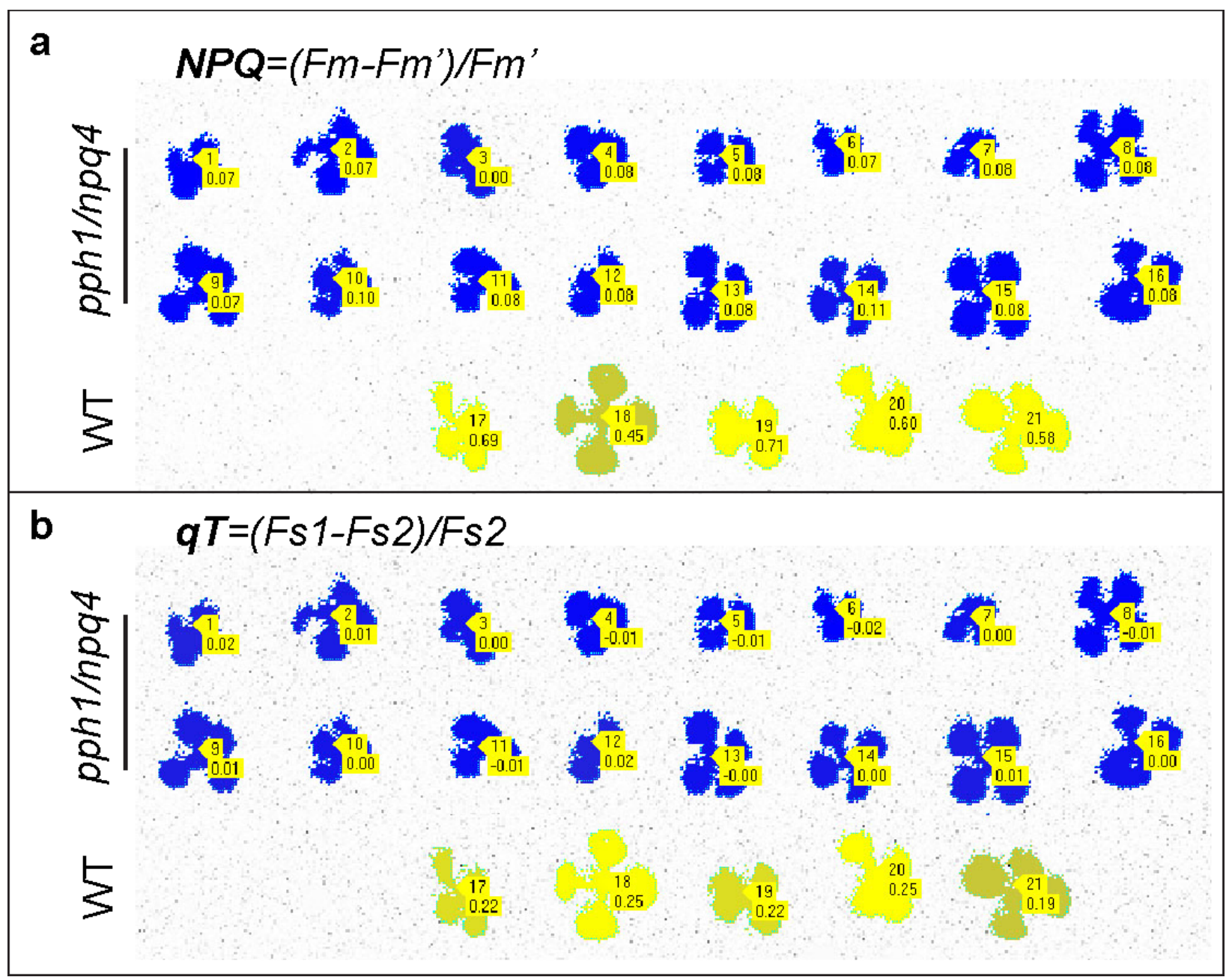

Figure S2. Beside experiments shown in Fig. 1, homozygosis of pph1/npq4 F3 plants has been further phenotypically confirmed by checking the decreased induction of NPQ and the lack of state transitions using an imaging PAM fluorometer. a For NPQ induction, a very short time $(45 \mathrm{~s})$ and weak light (40 $\mu$ mol photons $\left.\mathrm{m}^{-2} \mathrm{~s}^{-1}\right)$ have been used. With these conditions, a transient NPQ dependent on PsbS is clearly visible (Kalituho et al. 2007, Plant Physiol. 143:1861-70). b For State transitions, the parameter $\mathrm{qT}=(\mathrm{Fs} 1-\mathrm{Fs} 2) / \mathrm{Fs} 2$ is calculated and shown in the image. Fs1 is the fluorescence yield under actinic light after 15 minutes of State 1 induction and Fs2 is the fluorescence yield under actinic light after 5 minutes of State 2 induction (see Fig. 1) (Crepin and Caffarri 2015, Biochim Biophys Acta Bioenerg 1847:1539-48). 


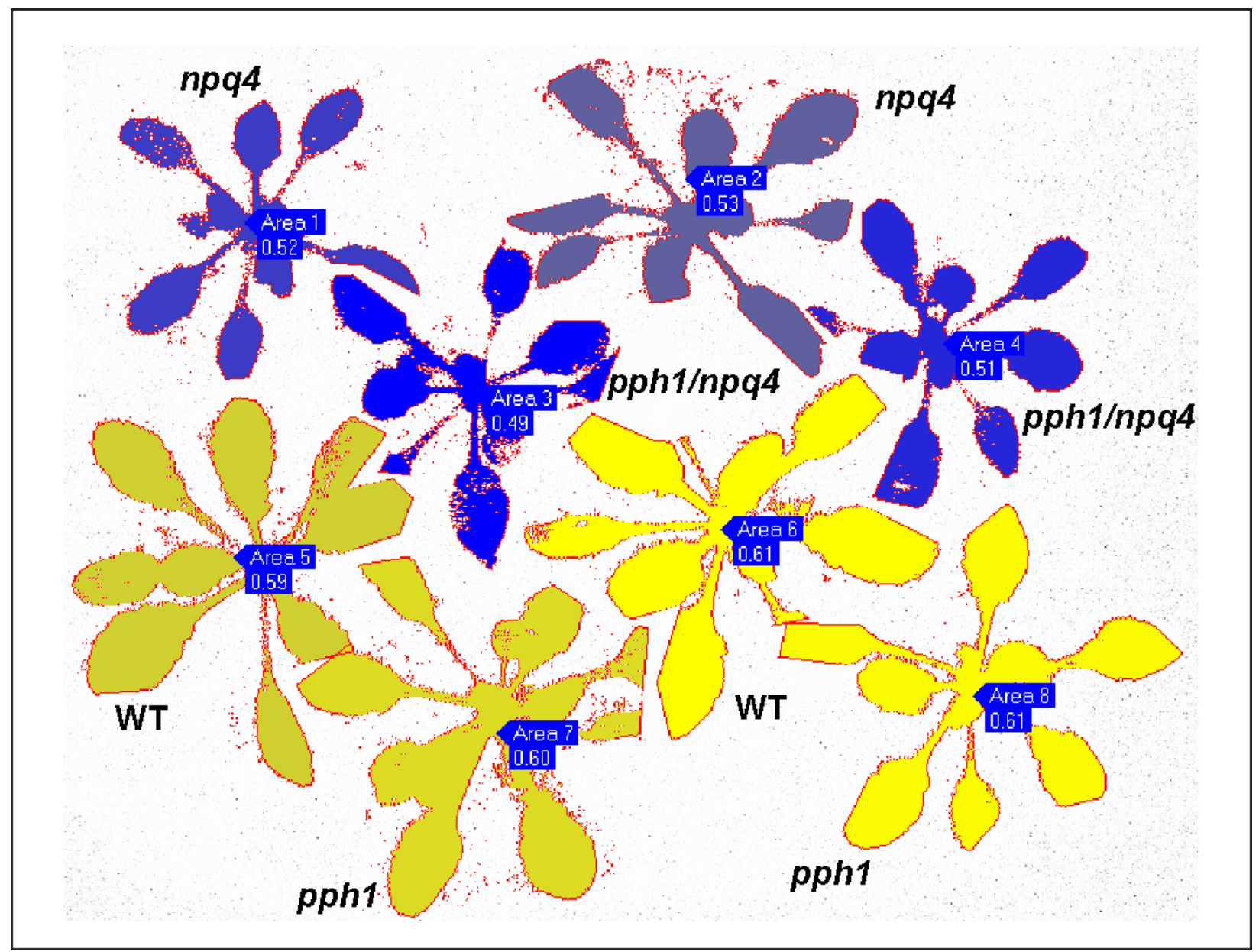

Figure S3. Fluorescence image of representative plants for the high light stress described in Fig. 2. The whole high light-exposed part of the plant has been used for the calculation of $\mathrm{Fv} / \mathrm{Fm}$ (the numbers indicated are the average values calculated on all pixels of each plant).

a

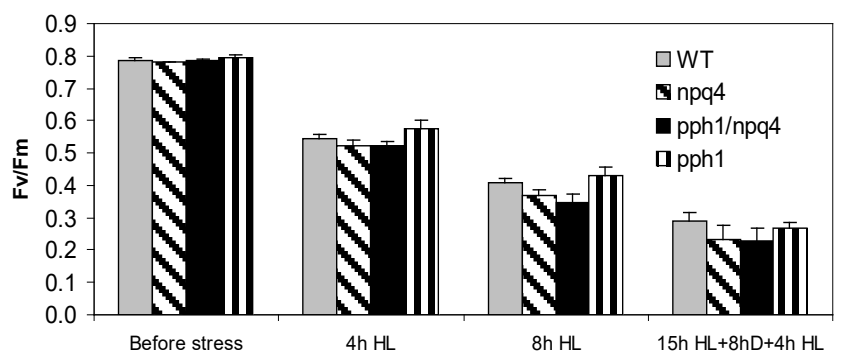

b

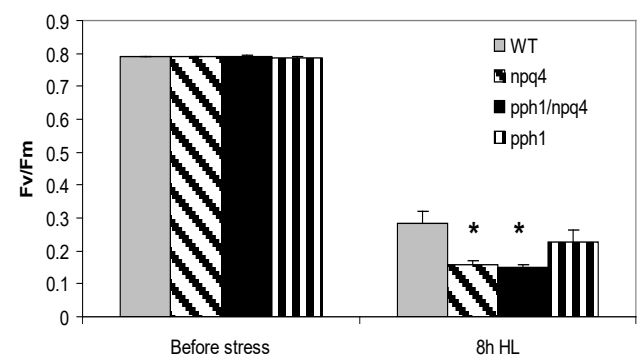

Figure S4. Fv/Fm measured before and after treatment by high light. a Plants grown 17 days in NL, then 2 months in LL were treated under $800 \mu \mathrm{mol} \mathrm{m} \mathrm{m}^{-2}$ for $4 \mathrm{~h}, 8 \mathrm{~h}$, and $15 \mathrm{~h}$ illumination plus $8 \mathrm{~h}$ in the dark followed by $4 \mathrm{~h}$ of illumination. $\mathrm{Fv} / \mathrm{Fm}$ values are the means and $\mathrm{SE}$ of measures on 3-4 plants for genotype, each plant measured on three different leaves with a dual PAM. P-values are $>5 \%$ for this test. b Fv/Fm before and after a treatment under $1500 \mu \mathrm{mol} \mathrm{m} \mathrm{m}^{-2}$ on plants similar as in panel a. Graph show the means and SE of measures on 3, 4, 7, 3 plants for wild type, $n p q 4$, $p p h 1$, pph1/npq4 genotypes, respectively, each plants measured on three different leaves. The $\mathrm{Fv} / \mathrm{Fm}$ decrease of $n p q 4$ and $p p h 1 / n p q 4$ compared with wild type is statistically significant with $\mathrm{p}$-value $<4 \%$ (* in figure), but difference is not statistically significant between wild type and $p p h 1$ ( $\mathrm{p}$-value $=25 \%$ ). 


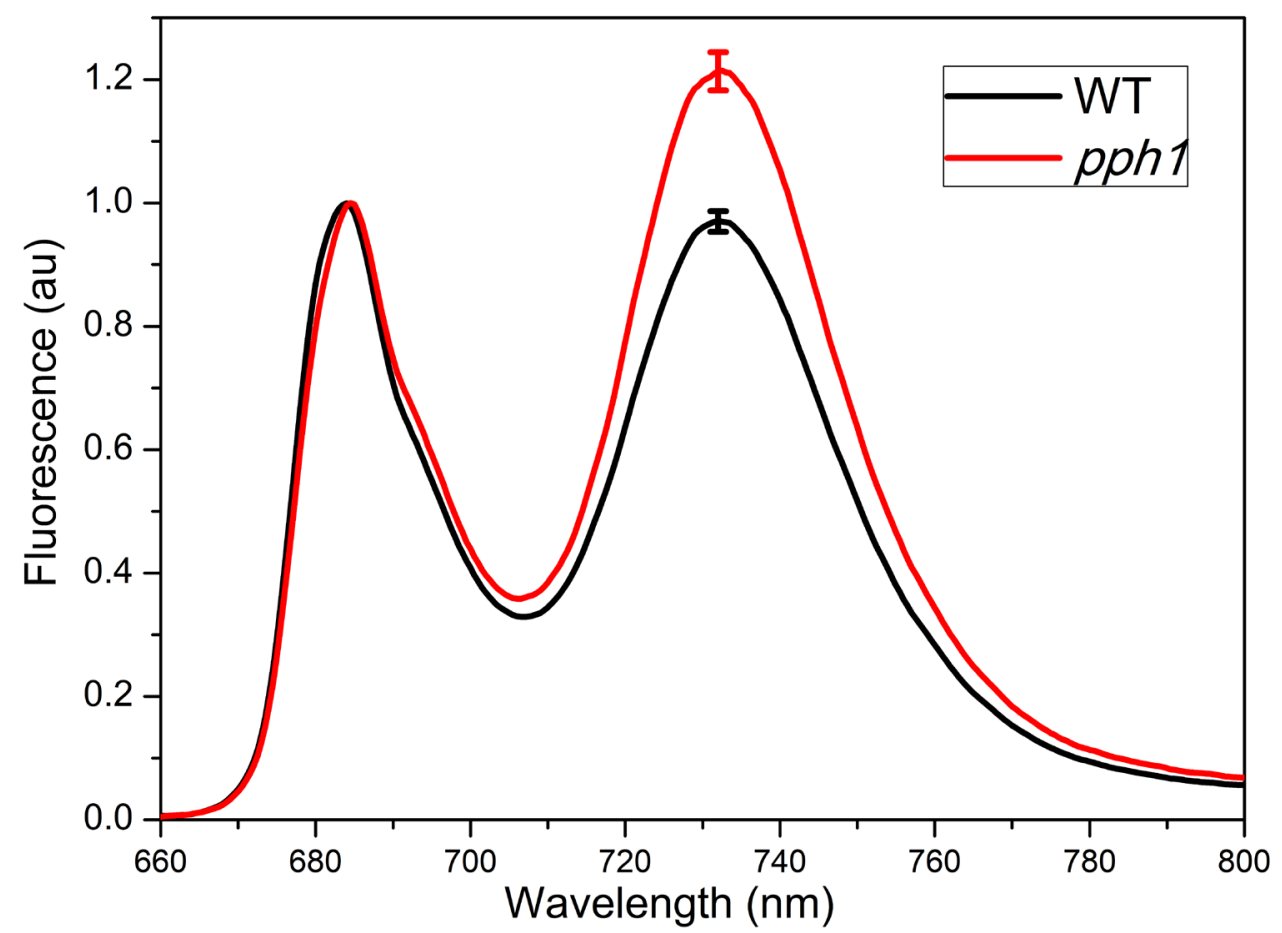

Figure S5. 77K fluorescence emission spectra of leaves of wild type and $p p h 1$ mutant plants after treatment at high light. Plants grown $\sim 2$ weeks in NL have been treated for 12 hours at $1500 \mu \mathrm{mol} \mathrm{m} \mathrm{s}^{-2}$ at $22^{\circ} \mathrm{C}$ and then frozen immediately after harvesting. Samples have been measured as indicated in the Material and Methods section. The spectra were normalized at the maximum PSII emission (peak at $684 \mathrm{~nm}$ ). Traces are the average of replicates on 6 independent plants for each genotype. SE at the maximum emission of PSI is indicated 